\title{
Remote Laboratory for Control Engineering Degree
}

\author{
Antoni Grau and Yolanda Bolea \\ Automatic Control Department, Technical University of Catalonia (UPC) \\ Pau Gargallo 5, 08028 Barcelona; e-mail: \{antoni.grau, yolanda.bolea\}@upc.edu
}

\begin{abstract}
This paper presents an innovative web-based control laboratory focused on the necessity of control education community. The usefulness of the remote laboratory proposed in this paper is justified by the opportunity of organizing the remote experiments, saving time and money to the students.
\end{abstract}

\section{INTRODUCTION}

In the present society, distance education offers a suitable solution to students that demand for an education system much more flexible, accessible and adapted (without spatial, temporal or economical restrictions). In overcrowded universities is not possible to offer enough laboratories to place all the students or give them specialized experimentation sites. The laboratories timetable is limited, normally, without the presence of an instructor, with its consequent under use. The acquisition and constant maintenance of laboratory equipment represent an excessive cost that can not be afforded for some colleges (Candelas et.al., 2005). The Engineering studies have high practical contents. Particularly, in control engineering the set of practices that make up the curriculum has the objective to illustrate the results obtained in the theoretical classes, as well as the student becomes familiar with equipment and instruments' manipulation in actual situations (Candelas et al.,2004). Therefore, it is important and necessary to move this practical environment to distance education through what is called remote laboratories, RemLab. To tackle the practices in control engineering the needed element is the existence of an automatic control education system consisting in a virtual laboratory (Grau et al., 2006) and telepresence accessible through a TCP/IP protocol-based network. This will allow students to practice in a more similar way as if they were in situ in the laboratory, giving the possibility to manage simulations and interactive software with the actual plants.

Thus, in a world with large evidences of the lack of resources and natural disasters, climate change... educational programs have to be developed adding especially sustainable concepts in all the educational levels (from primary school to university) for all the social groups. Furthermore, in these remote laboratories some habits, cognitive knowledge and values must be transmitted to future generations in order to have an influence in the attitudes and behaviour changes among the students that will be the future technical professionals.

\section{METHODOLOGICAL PROPOSALS}

In Control Engineering degree students will acquire knowledge, theoretical and practical abilities to automate and robotize systems and process, including analysis and design aspects, to increase performance, productivity, competitiveness, quality and to optimize energy and human resources in a broad range of sectors like for instance: industrial, aerospatial, naval, biomedical, natural and energy resources, services, among others.

General contents of this degree are: signal and systems theory; control engineering; mathematical methods; robot control and programming; systems modelling and simulation; sensors and perception systems; multivariable and non-linear control; advanced digital control; real time systems; industrial communications; hybrid systems; computational geometry in robotics; integrated production systems; mobile robots and teleoperation; artificial intelligence applied in control and robotics; pattern recognition; computer vision.

To reach the proposed goal, quality teaching and learning procedures using new technologies (digital campus, working groups, distance education, virtual and remote laboratories, etc.) will be used (Dormido, 2004) (Sánchez et al., 2005). This will allow the students to get a high level of knowledge and to be able to develop research tasks, either at the university or in industry.

It is proposed an innovative pedagogical methodology based on virtual and laboratory remotes where it is integrated greening and sustainable aspects overall in the practices. The course is divided into two parts:

1. Theoretical Concepts (according the subject of the degree) as master classes.

2. Practices by a versatile case-study plant with a specific simulation software (Easy Java Simulations, Ejs, introduced below) using virtual and remote laboratories.

To carry out the practices with virtual laboratories a CDROM, containing the Ejs software, and handbook as well as the practice statements, is sent to the students by mail. Students need to install and run Ejs in their own computers following the handbook explanations and developing later the virtual labs.

A remote laboratory is also proposed to the students as another material course, and it will be described in the next section. 


\section{DESIGN OF REMOTE LABORATORY SYSTEM}

There are today many technical universities that organize distance education. The courses and the simulation laboratories are distributed on electronic support, virtual laboratories and e-mail. However, the laboratory experiments that use physical equipment are not replaceable, because the student must have a direct contact with the real technical environment. Part of the work has to be organized in the institution, while the other part may be performed from the remote site through remote laboratories with Internet access to direct manipulation of real systems. The software to access to the remote plant is mainly a web browser and (in some cases) an application that can be downloaded from the laboratory server (Candelas et al, 2005). In the sequel, the structure of such a laboratory will be described. The following terms are used throughout the paper: the server is the computer situated in the laboratory, interfaced with the physical equipment; the client is the student's computer, remotely situated. A very general laboratory structure must include:

- The equipment (the regulated object, in the case of automatic control laboratory) associated with a computer server and a data acquisition card (in the case of virtual experimentation, the equipment is replaced by a simulation software).

- An interface program, running on the server computer, that drives the signals to and from the data acquisition card and manages the information about the student's work.

- Server software that deals with the communication between the laboratory and the remote students.

- The computer environment (usually Intranet and Internet) access through Internet or the campus backbone is necessary to the experimentation unit through the Remlab server.

- A client software running on the remote (student's) computer, that provides the Graphic User Interface and communicates with the server.

\subsection{Plant Description}

The nature of equipment depends on the subject of the laboratory. In our case, a unique plant for all subjects of Control Engineering degree has been selected. For this reason, the plant is a MIMO system that can be used to design SISO and MIMO system control as well as in subjects in B.S. degree or M.S. degree. The plant can also be used in post-graduated studies where more complex control techniques (no-linear control, robust control, coupling techniques...) are taught.

The plant consists of two tanks TT, TD with cross section A. The full structure of the plant is shown in Fig. 1.

The tanks are connected in serially with each other by pipes. On the right and the left side of the tanks TT and TD there are the pumps $(1,300 \mathrm{l} / \mathrm{h})$ and the single output valves $(0-$ $230 \mathrm{~V})$. The output-flowing liquid of the TD is collected in a reservoir located under the two tanks. The reservoir supplies flow to TT by a pump $(3,800 \mathrm{l} / \mathrm{h})$ and an output valve $(0$ $230 \mathrm{~V})$. In fact, the plant is a closed system, where the liquid that arrives to the reservoir from the tanks returns to the tanks via the pumps.

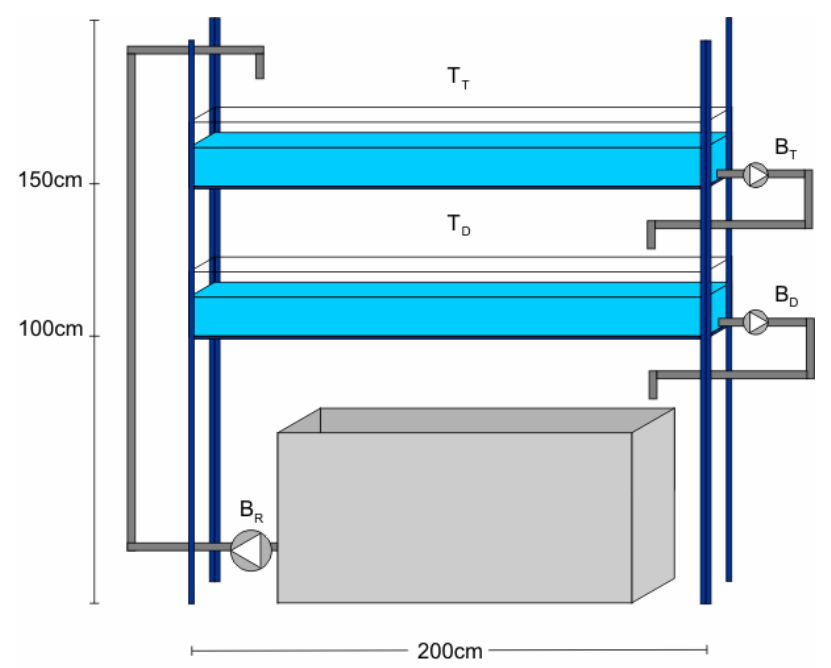

Fig.1. Full structure of plant

\subsection{Control Software}

In control engineering, the relationships among the variables of a system are accurately and clearly defined by mathematical language, but its visualization is complicated for students. This is not a simple mental exercise. It is true that there are many mathematical packages to program these relationships and thus to observe the results of changing some parameters of the system, but these tools are mainly oriented towards a non-interactive approach composed of two stages: design and analysis. That is, students have to afford many iterations with the tool to appreciate the level and sense of the change in some parameters. Moreover, such a static approach does not always facilitate the global view of the relationships among the variables of the system. The amount of data that a static approach can produce and the number of iterations needed to obtain them makes it difficult to students to build a mental scheme of the interactions among the components of the system. In these cases, a qualitative and conceptual view of the system can be a better approach to lead students to a deeper understanding of the system.

However, the control engineering teacher is an expert in his/her subject but not in programming techniques. The development of interactive didactic applications is not so easy with the typical tools that a control engineering teacher uses to explain concepts, as for example, Matlab/Simulink. At the same time, there are many commercial tools designed to create animations and multimedia but, in general, scientific simulations can not be created with them because they make a poor or inexistent use of the mathematical language to 
define the system to model. Easy Java Simulations (EjS) is a tool that can be the first in the colonization of this no-man's land. It should be considered as a first step in the development of further and more advanced tools intended for control engineering educators with some knowledge of programming, not for advanced programmers with little knowledge of control and modelling. Ejs has been conceived for people who are more interested in the content of the simulation, the simulated phenomenon itself, and much less in the technical aspects needed to build the simulation. Hence, EjS provides a conceptual structure and simplified tools that allow concentrate most of the time in the description of the model of the system to simulate. To help assist educators in the field of control engineering, Ejs has been extended so that it can be used in conjunction with Matlab/Simulink, a de facto standard in control engineering. Inspired in the model view-control software paradigm, Ejs facilitates the creation of the user interface, and Matlab/Simulink lets teachers develop the model using the language they are familiar with. Ejs is a freeware, opensource tool developed in Java, specifically designed to create interactive dynamic simulations (url1). The architecture of Ejs derives from the model-view control paradigm, whose philosophy is that interactive simulations must be composed of three parts: the model, the view, and the control. According to that, the steps to build an application in Ejs are the following: (1) To define the model is necessary to specify the variables that describe the system and the mathematical equations interrelating them; (2) define the view in order to represent the states of the process; and (3) define the control in order to describe the actions that the modeler can execute above the simulation.

Once the modeler has defined the model and the view of the interactive simulation, Ejs generates the Java source code of the simulation program, compiles the program, packs the resulting object files into a compressed file, and generates HTML pages containing the narrative and the simulation as an applet. The user can readily run the simulation and/or publish it on the Internet. Developers can then insert Matlab code in an Ejs application to make calculations in the Matlab workspace. But also, the model of a system can be completely or partially developed using just Simulink block diagrams. Through the applet, students can access to the sliders in order to change some variable values. Ejs collects these new values and pass them to Simulink. In this moment, the control over the plant can be modified and, therefore, the plant behaviour can change according to the sliders values.

After examining the state of the art in other disciplines, it is clear that an exciting task must be accomplished to improve the computer learning experience in the control engineering curriculum. New interactive modules for concepts in control systems have to be developed, and new tools must arise. From the experience obtained with the creation of the first such ones, new ideas to design the second generation must flourish.

The server has been developed with Tomcat web server, and we have implemented some web services to remotely access to Matlab instead of using the Matlab Web Service, which has demonstrated to not work properly, Fig. 2. With this toolbox (Web Service) is not possible to execute all the Matlab functions. To monitor the plant behaviour an IP camera has been set up.

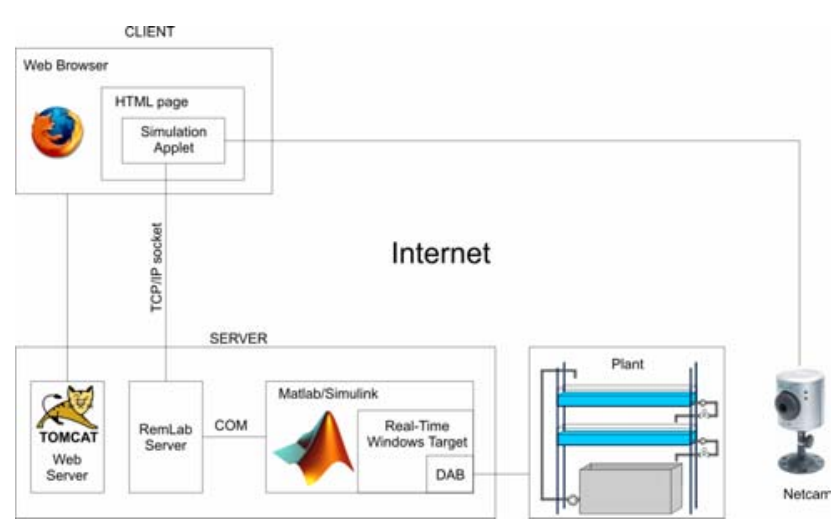

Fig.2. Software architecture

The control of the plant is done by Simulink through an IO card that access to the electrovalves to control the flow and reads the level sensor values. The RemLab Server is an application developed in Visual Basic that has the ability of reading and writing values to/from Simulink. The read values are the level sensor values and they are sent to the Java applet through the socket. In a similar way, the applet sent to the server the level setpoint that is updated in the Simulink. At the same time, in the client part, the applet is doing two tasks: first, the received values from the real control are plotted in order to remotely observe the control evolution; second, the applet runs the simulation of the real plant, in order to verify that the model coincides with the plant, the simulated values are plotted together with the real ones. Thanks to a webcam, it is possible to see the real evolution through the images and overlap the simulation evolution view. This effect is quite astonishing for the students demonstrating the power of EjS to view and represent simulation results.

\section{PRACTICES OF COURSE}

Students in control engineering education should achieve knowledge and skills of control systems modelling in order to develop controllers that enforce performance requirements. Once a controller is designed and implemented, observation of the resulting dynamics gives valuable insight into design concepts. Making use of web-based laboratories, students can observe dynamic phenomena that are often difficult to explain in written form.

Interactive experimentation on real world plants improves the motivation of students and also develops an engineering approach to solve realistic problems. Simulation is a proper way to complement control education but in general, it cannot replace the experimentation on real plants, since a simulation is only as good as the model is, and a model is just an approximation which cannot reproduce every aspect of the process, such as, for instance, unexpected non-linearities. 
In a remote web-based laboratory, students can access to the lab from a remote location that allows them to interact with real experiments. They can change control parameters, run experiments, see the results, and download data through the web (url2). Furthermore, an experimental approach to explain the most usual control system benchmarks could indeed improve the motivation of students, avoiding to be constrained just to look at simulations as results of their work previously simulated in a virtual laboratory.

At the beginning of the semester each student is provided with a password to authenticate him/her in the web page. This password is validated against the database (DB). Practice instructors schedule the use of the plant and each student has assigned a time slot. During this time he/she has the plant in mutual exclusion. Although the existence of this scheduling policy, there are free slots and the student that access to the plant has a limited time of usage.

In this context, the two-tank system can be proposed as casestudy system for different purposes, as test system for fault detection and identification, as well as for advanced control. It has received a great attention because it presents interesting properties in both control education and research. The system has been made for to serve as a test-bed for algorithms related to state estimation, and control or identification of hybrid systems. For these reasons, the two-tank system is used to show the results of different control strategies and as an educational tool in teaching advanced control techniques in Technical University of Catalonia (UPC).

Issues of possible practices:

1. Modelling and simulation of a tank. Simulation of the tank model behaviour and comparative with real tank behaviour.

2. Modelling and simulation of two serial tanks. Simulation of the behaviour of the obtained MIMO model tank and comparison with dynamics of the real tank system.

3. Identification of the system by the obtained input/output data plant, as in the SISO case as well as in the MIMO case.

4. Control of the tank by classical controllers (PI, PID) with simulation and remote experiments.

5. Control of the serial tanks by different methods of advanced control: optimal control, robust control, etc.

6. Control of the serial canal system by the Smith Predictor configuration. This practice implies a slight modification of the tanks, introducing some methacrylate sheets in order to create a larger path for water. Specifically, with sheets located at $4 \mathrm{cmts}$ of distance, the path increases its length from $2 \mathrm{mts}$ to $14 \mathrm{mts}$.

Next, we describe a proposed practice with the previous plant.

\section{EXAMPLE OF AN INTERACTIVE CONTROL PRACTICE}

\subsection{Statement}

This section describes a control experience for the basic subjects in control engineering degree. The objective is to perform a single tank (TD) level control fulfilling the control specifications. Therefore, the tank level is regulated by the reservoir electric pump (BT) and its output valve (for tank filling), as well as the tank electric pump (BD) and valve (for tank emptying), see Fig. 1. This operation implies an MISO control, but only a single controller is required (an SISO controller) switching between both control signals (BT and $\mathrm{BD}$, i.e. filling and emptying) according to the required actuation to achieve the set-point level (h2).

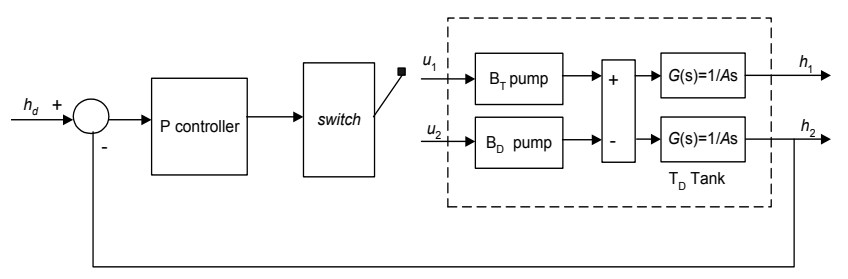

Fig. 3. Block diagram for the control system of the TD tank.

The imposed control specifications for step set points are: stability, permanent zero-error, and full operating range for electric valves (from 0 to 230 volts). For this purpose a $\mathrm{P}$ (proportional) controller is needed because the plant model has already an integrator warranting a permanent zero-error for step reference signals.

Fig. 3 shows a block diagram of the implemented control system to regulate the level tank TD. In Fig. 4 it can be observed the evolution of the controlled variable (TD level, $h 2$ ), the set point or desired level $(h d)$ and the control variables $u 1$ and $u 2$, that is, the input voltages of the BT and BD pumps, respectively.

\subsection{Pilot Study and Evaluation Results}

To assess the objectives and goals that were set in this training experiment, a methodological process was followed, based on a specially designed experimental evaluation protocol from a pedagogical perspective.

From this approach, the goal is to assess the performance of such e-laboratory in terms of "quality" in the following aspects: 1) these subjects can be within everyone's reach vs. the "traditional subjects" (space, material, resources and timetable), 2) training is tested mainly according three important characteristics (building awareness, knowledge and skills).

With respect the former point, the distance education offers the possibility that the practices of these experimental subjects can be taken by any profile of student. The benefits from providing the means to obtain remote access to 
experimental infrastructure existing in various dispersed laboratory facilities can become significant both from a socioeconomic point of view, as well as from an educational perspective.

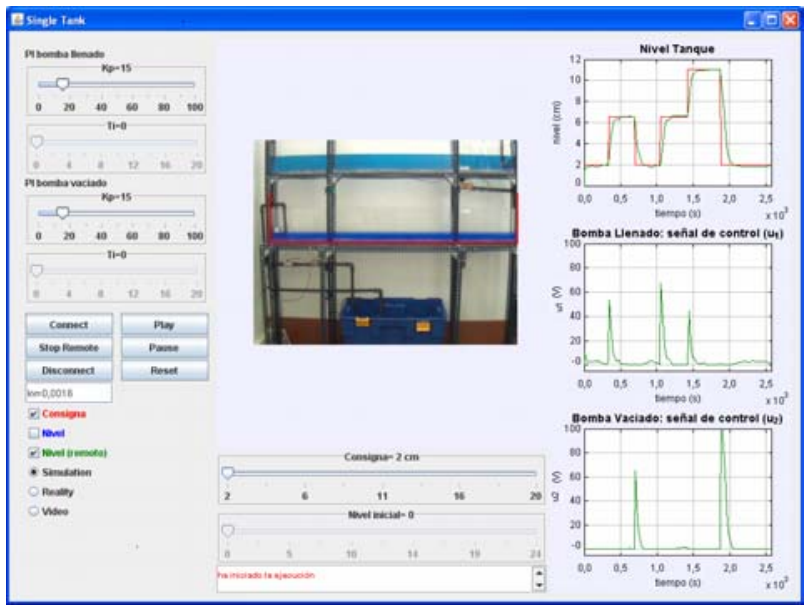

(a)
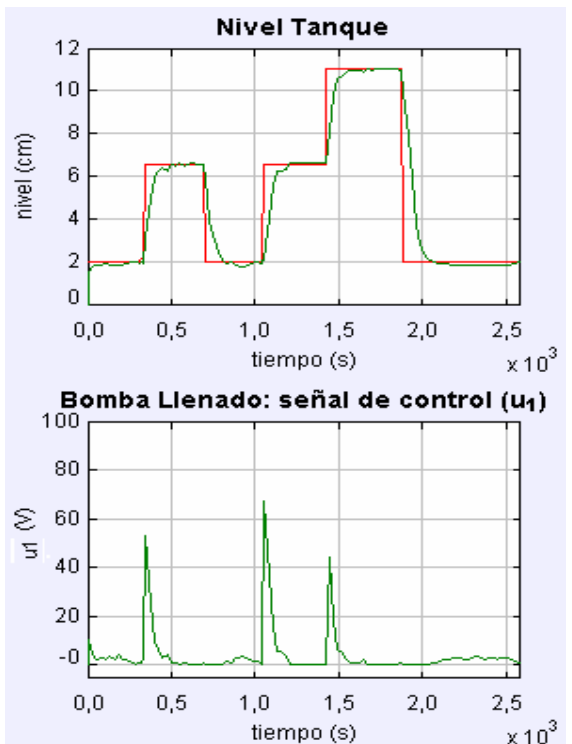

Bomba Vaciado: señal de control $\left(u_{2}\right)$

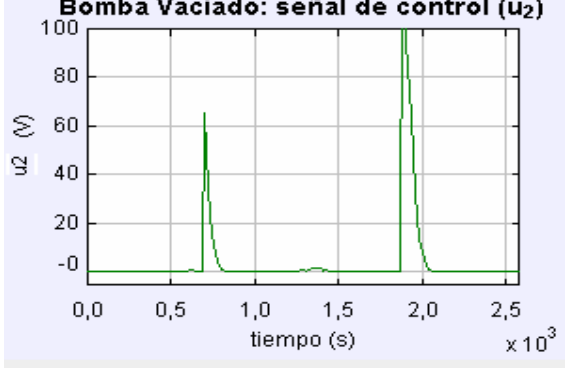

(b)

Fig. 4. (a) View of the remote control of the TD tank of the pilot plant and evolution of the controlled and manipulated variables of the tank process;

(b) enlargement of the evolution of these variables.

This significance is directly related to the quality and the equity of practical training possibilities offered to all students. In this context, a more thorough experimental evaluation study has to be conducted, regarding the feasibility of these goals and the acceptability of such new technologies by students in their education and training practice.

The latter point has been tested by a pilot study of a laboratory training course of 30 students where the obtained results validate some advantages of this training process versus the traditional methods such as improvement of the learning by the reach of the best high-level skills and understanding. The indicators to check the quality of the learning are mainly opinion polls to the students and their qualifications during the semester.

\section{CONCLUSIONS}

This paper describes the development of an e-learning laboratory platform in the field of control systems. A virtual laboratory oriented to control theory, automatic regulation, advanced control and modelling subjects in the Control Engineering Degree is presented. From a technological perspective, this work focuses on the adaptation of concepts and techniques developed in the field of engineering control and on exploring their integration in such remote laboratory settings. This lab is developed to apply the advantages in distance education in an engineering field where the real practices are relevant because with the unique use of simulation students are not suitable to verify the design results. The importance of this type of training is directly related to the quality and the equity of practical training possibilities offered to all students. Besides, through these remote laboratory practices instructors have detected a bigger learning capacity of the students as well as the enthusiasm of the practices realization.

\section{ACKNOWLEDGMENTS}

Authors thank the Inter-ministerial Commission of Spanish Government (DPI2004-5414, MCYT), the Catalan Autonomous Government (VIS, Consolidated Research Group) and the European Commission (FP6-2005-IST-6, URUS-045062) for funding the work.

\section{REFERENCES}

Candelas, F.A. and Sánchez, J., "Didactic resources on Internetbased in system engineering and automation", Latinamerican Journal on Automation and Industrial Informatics, vol. 2, no. 2, pp. 93-101, April 2005.

Candelas, F.A., Torres, F., Gil, P., Ortiz, F., Puente, S. and Pomares, J., "Virtual remote laboratory and its impact evaluation in Academics", Latinamerican Journal on Automation and Industrial Informatics, vol. 1, no. 2, pp. 49-57, July 2004.

Grau, A. and Bolea, Y. "Virtual Laboratories for Ecological Systems Modeling", International Workshop for Computer Education, SIIE, León, Spain, 2006.

Dormido, S., "Control Learning: Present and Future", Annual Reviews in Control, vol. 28(1), pp.115-136, 2004.

Sánchez, J., Dormido, S. and Esquembre, F., "The learning of control concepts using interactive tools", Comput. Appl Engineering Education, vol. 13, pp. 84-98, 2005.

url1:Easy Java Simulations' home page at http://fem.um.es/Ejs

url2: http://model.upc.edu 